\title{
Caracterização dos incidentes em um Hospital Público Federal entre os anos 2014 e
}

\section{9}

\author{
Characterization of incidents in a Federal Public Hospital between the years 2014 and 2019
}

Caracterización de incidencias en un Hospital Público Federal entre los años 2014 y 2019

Recebido: 19/03/2021 | Revisado: 26/03/2021 | Aceito: 02/04/2021 | Publicado: 12/04/2021

\author{
Alice Eulália Chagas Ribeiro \\ ORCID: https://orcid.org/0000-0001-6068-9354 \\ Universidade Federal do Estado do Rio de Janeiro, Brasil \\ E-mail: aliceeulalia@yahoo.com.br \\ Cristiane de Oliveira Novaes \\ ORCID: https://orcid.org/0000-0002-5272-3759 \\ Universidade Federal do Estado do Rio de Janeiro, Brasil \\ E-mail: novaes.cristiane@gmail.com
}

\begin{abstract}
Resumo
Introdução: a ocorrência de incidentes em serviços de saúde pode provocar graves repercussões ao paciente. A fração da mortalidade atribuível (FAP) de eventos adversos graves que são preveníveis foi 30,5\% no Sistema único de Saúde e 36,1\% no Sistema de Saúde Suplementar. Objetivo: caracterizar os incidentes de um hospital geral público federal de referência na Cidade do Rio de Janeiro no período entre janeiro de 2014 e dezembro de 2019. Método: estudo transversal, retrospectivo e documental, realizado a partir da análise de notificações. A coleta de dados foi realizada entre 10 e 19 de fevereiro de 2020. Resultados: foram notificados 10.832, no período entre 2014 e 2019. A caracterização dos incidentes foi classificada segundo as Metas Internacionais de Segurança do Paciente, Eventos Adversos, Incidentes sem Dano, Quase Falha, Circunstâncias Notificáveis e o item "Não se aplica". Considerações finais: assegurar a identificação, a prevenção e criar soluções para a redução de eventos adversos estabelecendo procedimentos de notificação, comunicação e envolvimento centralizado no paciente, são algumas das estratégias para melhorar a qualidade do cuidado e um passo para a cultura de segurança.
\end{abstract}

Palavras-chave: Segurança do paciente; Eventos adversos; Erros médicos; Metas de saúde.

\begin{abstract}
Introduction: the occurrence of incidents in health services can cause serious repercussions for the patient. The fraction of attributable mortality (FAP) of serious adverse events that are preventable was 30.5\% in the Unified Health System and $36.1 \%$ in the Supplementary Health System. Objective: to characterize the incidents of a reference public federal general hospital in the city of Rio de Janeiro in the period between January 2014 and December 2019. Method: cross-sectional, retrospective and documentary study, carried out from the analysis of notifications. Data collection was carried out between February 10 and 19, 2020. Results: 10.832 were reported in the period between 2014 and 2019. The characterization of the incidents was classified according to the International Patient Safety Goals, adverse events, incidents without damage, almost failure, notifiable circumstances and the item "not applicable". Final considerations: ensuring identification, prevention and creating solutions to reduce adverse events by establishing procedures for notification, communication and centralized patient involvement, are strategies to improve the quality of care and a step towards a safety culture.
\end{abstract}

Keywords: Patient safety; Adverse events; Medical errors; Health goals.

\section{Resumen}

Introducción: la ocurrencia de incidencias en los servicios de salud puede ocasionar graves repercusiones para el paciente. La fracción de mortalidad atribuible (PAF) de eventos adversos graves prevenibles fue del 30,5\% en el Sistema Único de Salud y del 36,1\% en el Sistema Complementario de Salud. Objetivo: caracterizar los incidentes de un hospital general público federal de referencia en la ciudad de Río de Janeiro en el período comprendido entre enero de 2014 y diciembre de 2019. Método: estudio transversal, retrospectivo y documental, realizado a partir del análisis de notificaciones. La recolección de datos se llevó a cabo entre el 10 y el 19 de febrero de 2020. Resultados: se reportaron 10.832 en el período entre 2014 y 2019. La caracterización de los incidentes se clasificó de acuerdo a las Metas Internacionales de Seguridad del Paciente, Eventos Adversos, Incidentes sin Daño, Casi Fallo , Circunstancias notificables y el ítem "No aplica". Consideraciones finales: asegurar la identificación, prevención y generar soluciones para la reducción de eventos adversos mediante el establecimiento de procedimientos de notificación, comunicación y participación centralizada del paciente, son algunas de las estrategias para mejorar la calidad de la atención y un paso hacia una cultura de seguridad.

Palabras clave: Seguridad del paciente; Eventos adversos; Errores médicos; Objetivos de salud. 


\section{Introdução}

A Organização de Cooperação e Desenvolvimento Econômico (OCDE) aponta que 15\% dos custos hospitalares são referentes à ocorrência de eventos adversos, e que o custo total derivado desse quadro esteja na ordem de trilhões de dólares por ano (Slawomirski, 2017). Somado a isso, a incidência de óbito ultrapassou a taxa de $20 \%$ nos pacientes vítimas de eventos adversos graves. Os principais eventos relatados foram, a endocardite infecciosa, septicemia, choque séptico, úlcera por pressão, insuficiência respiratória aguda, aspiração pulmonar, mediastinite, insuficiência renal aguda, pneumonia, meningite e infecção relacionada a dispositivo intravascular. A fração atribuível populacional (FAP) mede o efeito da eliminação do fator de risco (eventos adversos) para a diminuição da mortalidade, foi 30,5\% no SUS e 36,1\% no Sistema de saúde Suplementar (Couto, 2018).

Por essa razão, em anos mais recentes, o cuidado seguro tornou-se uma prioridade e tem consumido esforços em nível mundial, em especial a partir da publicação do relatório de Harvard "To Err is Human”, que tornou-se referência mundial na área, publicado pelo Institute of Medicine (IOM, 2001). Nesse campo a World Health Organization (WHO) criou, em 2004, o programa The World Alliance for Patient Safety, com o objetivo de desenvolver políticas mundiais para o cuidado aos pacientes nos serviços de saúde, e entre as iniciativas desse programa, destaca-se o desenvolvimento de uma taxonomia, que define o termo "incidente com dano", sinônimo de evento adverso (Mendes, 2019). Nesse caso, entende-se por evento adverso ou incidente com dano, o prejuízo não intencional causado ao paciente, não relacionado à morbidade de origem, em decorrência das intervenções da equipe de saúde que pode gerar prolongamento do tempo de internação, sofrimento, desconforto físico e emocional, incapacidade e óbito (WHO, 2012).

Diante da magnitude do problema em termos de risco, frequência e ocorrências dos eventos adversos, a WHO (2004) visando a redução da ocorrência de incidentes nas áreas consideradas mais críticas da assistência, e assim maior qualidade do cuidado em serviços de saúde, estabeleceu seis metas internacionais, a saber: Meta 1 - Identificação correta dos pacientes; Meta 2 - Melhorar a comunicação entre os profissionais; Meta 3 - Melhorar a segurança na prescrição, no uso de administração de medicamentos; Meta 4 - Assegurar cirurgia em local de intervenção, procedimentos e pacientes corretos; Meta 5 - Higienizar as mãos para evitar infecções; Meta 6 - Reduzir o risco de quedas e úlceras de pressão.

No Brasil, as iniciativas envolveram a criação de mecanismos na legislação, como a Portaria no 1660 do Ministério da Saúde, de 22 de julho de 2009, que instituiu o Sistema de Notificação e Investigação em Vigilância Sanitária - VIGIPOS, no âmbito do Sistema Nacional de Vigilância Sanitária buscando monitorar os incidentes com dano e queixas técnicas referentes a produtos (VIGIPOS, 2009). Posteriormente, a ANVISA, por meio da Resolução da Diretoria Colegiada (RDC) nº 63, de 28 de novembro de 2011, estabeleceu padrões para ampliar as ações de segurança e qualidade ao instituir os Requisitos de Boas Práticas de Funcionamento (BPF) em saúde (ANVISA, 2011). Mais recentemente, a Portaria no 529, de 1o de abril de 2013, do Ministério da Saúde criou o Programa Nacional de Segurança do Paciente (PNSP), apresentando como objetivos: qualificar o cuidado, promover e apoiar a segurança do paciente nas áreas de atenção, gestão de serviços, implantação da gestão de risco e de Núcleos de Segurança do Paciente, envolver pacientes e familiares no cuidado, facilitar o acesso às informações sobre a segurança do paciente, disseminar conhecimentos e incluir a segurança do paciente no ensino, técnico, na graduação e pós-graduação na área da saúde (PNSP, 2013).

A vigilância pode ser vista como etapa fundamental a esses objetivos e, nesse sentido, a notificação dos eventos, além de obrigatória e regulamentada, propicia o monitoramento não só da ocorrência dos mesmos, mas de sua caracterização, subsidiando ações que contribuem para a identificação das causas e consequentemente da melhoria dos processos assistenciais, implicando em maior qualidade e segurança do paciente.

Sendo assim, a partir desse contexto, o presente artigo teve como objetivo caracterizar a ocorrência de incidentes em um Hospital Público Federal no Rio de Janeiro, no período de 2014 a 2019, com o intuito de identificar a magnitude do 
problema da segurança do paciente, criando subsídios para o manejo adequado do problema afim de contemplar a ampliação da segurança do paciente na Instituição.

\section{Metodologia}

Trata-se de um estudo quantitativo, transversal, retrospectivo, documental, a partir de dados secundários de acesso restrito e sem identificação dos pacientes e/ou profissionais envolvidos, realizado em um Hospital Geral Público da Rede Federal, referência em tratamentos clínicos e cirúrgicos, com 26 especialidades, 201 leitos, e cuja produtividade tem sido em torno de vinte mil consultas e quinhentas cirurgias por mês, localizado na Área Programática 2.1, na divisão administrativa de planejamento no Rio de Janeiro. De acordo com Pereira et al. (2018), nas pesquisas com métodos quantitativos, faz-se a coleta de dados quantitativos ou numéricos nas suas respectivas unidades, que por sua vez geram conjuntos de dados passíveis de serem analisados por meio de técnicas matemáticas.

A amostra compreendeu todos os dados referentes aos incidentes ocorridos entre janeiro de 2014 a dezembro de 2019 e o número de pacientes internados por ano. Cabe destacar, que de 2011 a 2013 os incidentes eram notificados por meio de formulário de ocorrências assistenciais e administrativas da instituição através de memorandos, ofícios, impressos depositados em urnas e via e-mails, e que apenas a partir da RDC 36 em 2013, tornou-se obrigatória as notificações de incidentes enviadas para o Núcleo de Segurança do Paciente (NSP). Não foram estipulados critérios de exclusão, tendo sido incluídos e analisados todos os incidentes notificados no período do estudo.

Os incidentes foram classificados nas seguintes subdivisões:

- As Seis Metas Internacionais de Segurança do Paciente;

- Adicionada a meta 7, para classificar úlceras de pressão, separada da meta 6;

- Item "não se aplica" para aqueles que não se aplicam às metas internacionais;

- Evento adverso equivalente a incidentes com dano;

- Incidentes sem dano;

- Circunstâncias Notificáveis considerados os fatores que podem influenciar um evento;

- Quase falha definido como o evento que não atingiu o paciente.

A análise estatística destinou-se a examinar a distribuição dos eventos por meio de análise univariada, para análise da distribuição da frequência, foi usado o software SPSS 18.0.

\section{Resultados}

Após o levantamento e extração dos dados das diferentes formas de notificação ou registro dos eventos, foram identificados, entre janeiro de 2015 e dezembro de 2019, um total de 47.202 pacientes internados. Chama atenção que o número de internações apresentou um discreto aumento progressivo ao longo do período. $\mathrm{O}$ total de incidentes foi 5488 entre os anos de 2014 e 2019, sendo que o ano com maior ocorrência foi 2016 com 1359 registros, seguido do ano de 2017 com 1273 registros. Em 2014 foram identificados 30 registros, a menor proporção identificada, o que pode ser explicado pelo contexto histórico em relação as ações de vigilância de eventos, que vem sendo priorizados em anos mais recentes. No caso do Hospital, local do estudo, as ações destinadas a segurança do paciente tiveram inicio de forma mais efetiva, após a constituição do Núcleo de Segurança do Paciente (NSP) criado pelo setor de Qualidade. O NSP foi nomeado e instituído em 2013, conferindo aos membros autoridade, responsabilidade e poder para executar as ações do PNSP e descrever estratégias e ações de gestão de risco, conforme as atividades desenvolvidas pelo serviço de saúde. 
A Tabela 1 mostra a análise da ocorrência e dos fatores associados aos Incidentes por Metas, podemos identificar um total de 5488 registros para esse campo, sendo que a maior parte das ocorrências referem-se à Meta 1, relacionada a identificação correta do paciente, com um total de 3.636 notificações, correspondente a 66,20\% das notificações e 7,07\% das internações totais do periodo. A falha na identificação correta do paciente (Meta 1) pode levar à graves consequências por atravessar todos os demais processos do cuidado, desde a internação até a alta. A identificação deve ser verificada antes de realizar procedimentos clínicos, cirúrgicos, hemotransfusões, realizar exames, coletar amostras de material, por exemplo.

Em segundo lugar, foram registradas 740 notificações para a Meta 5, que trata da prevenção de infecções, com percentual de 13,47\% dos incidentes por meta e a 1,57\% nas internações. A Meta 5 trata da prevenção de infecções relacionadas à assistência em serviços de saúde (IRAS). O serviço de Controle de Infecção Hospitalar (SCIH) acompanha os casos de infecção e participa em reuniões destinadas ao tratamento adequado ao paciente que adquire infecção durante a internação. A adesão ao processo exige um conjunto de ações que garantem o cuidado seguro, por meio de treinamento profissional na higienização das mãos: antes de tocar o paciente; antes de realizar procedimentos; antes de manusear um dispositivo; ao se mover de um sítio contaminado para outro do mesmo paciente; após o risco de exposição a fluidos corporais, excreções, mucosas, pele não íntegra ou curativo; após remover luvas esterilizadas ou não; após tocar o paciente antes e depois do contato com o paciente; apos remover luvas esterilizadas ou não; após tocar superfícies próximas ao paciente; após contato com superfícies e objetos inanimados.

A categoria com menos registros foi a Meta 4, com 31 registros que correspondem a $0,56 \%$ dos acidentes por meta e $0,06 \%$ das internações. Foi observado que em relação à Meta 4 não houve adesão à lista de verificação de segurança nas cirurgias. Esse processo exige perfeita comunicação, colaboração e harmonia de toda a equipe profissional para confirmação de dados do paciente, da cirurgia, da equipe médica do serviço de anestesia e da cirurgia, técnicos de enfermagem e enfermeiros, equipe da central de material e esterilização e responsáveis pelo transporte do paciente para a enfermaria, centro de tratamento intensivo (CTI) e unidade do pós-operatório. 
Tabela 1 - Ocorrência e Fatores Associados aos Incidentes: Análise por Metas.

\begin{tabular}{|c|c|c|c|c|c|c|c|c|c|c|c|c|c|c|}
\hline \multirow{2}{*}{ Classificação } & \multicolumn{2}{|c|}{2014} & \multicolumn{2}{|c|}{2015} & \multicolumn{2}{|c|}{2016} & \multicolumn{2}{|c|}{2017} & \multicolumn{2}{|c|}{2018} & \multicolumn{2}{|c|}{2019} & \multicolumn{2}{|c|}{ Total } \\
\hline & $\mathbf{N}$ & $\%$ & $\mathbf{N}$ & $\%$ & $\mathbf{N}$ & $\%$ & $\mathbf{N}$ & $\%$ & $\mathbf{N}$ & $\%$ & $\mathbf{N}$ & $\%$ & $\mathbf{N}$ & $\%$ \\
\hline $\begin{array}{c}\text { Total de } \\
\text { Pacientes } \\
\text { Internados }\end{array}$ & 5.941 & 100 & 6.471 & 100 & 6.845 & 100 & 6.951 & 100 & 10.070 & 100 & 10.924 & 100 & 47.202 & 100 \\
\hline $\begin{array}{c}\text { META 1 } \\
\text { Identificação } \\
\text { do paciente }\end{array}$ & 4 & 0,07 & 979 & 15,13 & 1.186 & 17,33 & 878 & 12,63 & 266 & 2,64 & 323 & 2,96 & 3.636 & $66,20 *$ \\
\hline $\begin{array}{c}\text { META 2 } \\
\text { Comunicação } \\
\text { efetiva }\end{array}$ & 1 & 0,02 & 30 & 0,46 & 25 & 0,37 & 28 & 0,40 & 55 & 0,54 & 53 & 0,49 & 192 & $3,50 *$ \\
\hline $\begin{array}{c}\text { META 3 } \\
\text { Utilização de } \\
\text { medicamentos }\end{array}$ & 2 & 0,03 & 27 & 0,42 & 56 & 0,82 & 39 & 0,56 & 39 & 0,39 & 41 & 0,38 & 204 & $3,71 *$ \\
\hline $\begin{array}{c}\text { META 4 } \\
\text { Cirurgia } \\
\text { Segura } \\
\end{array}$ & 3 & 0,05 & 2 & 0,03 & 2 & 0,03 & 14 & 0,20 & 7 & 0,07 & 3 & 0,03 & 31 & $0,56^{*}$ \\
\hline $\begin{array}{c}\text { META 5 } \\
\text { Prevenção de } \\
\text { Infecções }\end{array}$ & 0 & 0 & 119 & 1,84 & 0 & 0 & 161 & 2,32 & 200 & 1,99 & 260 & 2,38 & 740 & $13,47 *$ \\
\hline $\begin{array}{c}\text { META } 6 \\
\text { Quedas }\end{array}$ & 9 & 0,15 & 29 & 0,45 & 23 & 0,34 & 58 & 0,83 & 54 & 0,54 & 47 & 0,43 & 220 & $4,00 *$ \\
\hline $\begin{array}{c}\text { META } 7 \\
\text { Úlceras de } \\
\text { Pressão } \\
\end{array}$ & 4 & 0,07 & 26 & 0,40 & 25 & 0,37 & 21 & 0,30 & 0 & 0 & 1 & 0,01 & 77 & $1,40 *$ \\
\hline Não se Aplica & 7 & 0,12 & 48 & 0,75 & 42 & 0,61 & 74 & 1,06 & 58 & 0,58 & 159 & 1,46 & 388 & $7,07 *$ \\
\hline $\begin{array}{l}\text { Notificações } \\
\text { por Pacientes } \\
\text { Internados }\end{array}$ & 30 & 0,51 & 1.260 & 19,47 & 1359 & 19,85 & 1.273 & 18,31 & 679 & 6,74 & 887 & 8,12 & $5.488^{*}$ & $100 *$ \\
\hline
\end{tabular}

Fonte: Autores (2020).

Na Tabela 2 é apresentada a distribuição por tipo de ocorrência, com predominância dos eventos classificados como incidente sem dano com 3.029 registros, o que corresponde a 56,68\% dos incidentes por tipo de ocorrência e a $6,42 \%$ do total de internações. É provavel que os profissionais sintam-se mais seguros ao notificar incidentes sem dano por falta de confiança ou medo de punições. A notificação é válida porque um incidente sem dano pode levar ao dano /quase falha. A segunda classificação com mais registros foi a de evento adverso com 1.141 registros, correspondente a 21,33\% do total e a 2,42\% do total de internações, configurando efetivamente um incidente com dano ao paciente, durante a internação, sem relação com a patologia de base que o levou para o tratamento. 
Tabela 2 - Ocorrência e Fatores Associados aos Incidentes: Análise por Tipo de Ocorrência.

\begin{tabular}{|c|c|c|c|c|c|c|c|c|c|c|c|c|c|c|}
\hline \multirow{2}{*}{ Classificação } & \multicolumn{2}{|c|}{2014} & \multicolumn{2}{|c|}{2015} & \multicolumn{2}{|c|}{2016} & \multicolumn{2}{|c|}{2017} & \multicolumn{2}{|c|}{2018} & \multicolumn{2}{|c|}{2019} & \multicolumn{2}{|c|}{ Total } \\
\hline & $\mathbf{N}$ & $\%$ & $\mathbf{N}$ & $\%$ & $\mathbf{N}$ & $\%$ & $\mathbf{N}$ & $\%$ & $\mathbf{N}$ & $\%$ & $\mathbf{N}$ & $\%$ & $\mathbf{N}$ & $\%$ \\
\hline $\begin{array}{c}\text { Total de } \\
\text { Pacientes } \\
\text { internados }\end{array}$ & 5.941 & 100 & 6.471 & 100 & 6.845 & 100 & 6.951 & 100 & 10.070 & 100 & 10.924 & 100 & 47.202 & 100 \\
\hline $\begin{array}{l}\text { Evento } \\
\text { Adverso }\end{array}$ & 19 & 0,32 & 50 & 0,77 & 40 & 0,58 & 260 & 3,74 & 315 & 3,13 & 456 & 4,17 & 1.140 & $21,33^{*}$ \\
\hline $\begin{array}{c}\text { Incidente Sem } \\
\text { Dano }\end{array}$ & 4 & 0,07 & 843 & 13,03 & 1.202 & 17,56 & 724 & 10,41 & 145 & 1,44 & 111 & 1,02 & 3.029 & $56,68^{*}$ \\
\hline $\begin{array}{c}\text { Circunstâncias } \\
\text { Notificáveis }\end{array}$ & 3 & 0.05 & 223 & 3,45 & 75 & 1,09 & 110 & 1,58 & 186 & 1,85 & 286 & 2,62 & 883 & $16,52^{*}$ \\
\hline Quase Falha & 0 & 0 & 134 & 2,07 & 40 & 0,58 & 65 & 0,94 & 36 & 0,36 & 17 & 0,16 & 292 & $5,46^{*}$ \\
\hline $\begin{array}{c}\text { Notificações } \\
\text { por Pacientes } \\
\text { Internados }\end{array}$ & 26 & 0,44 & 1250 & 19,32 & 1357 & 19,82 & 1159 & 16,70 & 682 & 6,77 & 870 & 7,96 & $5.344^{*}$ & $100^{*}$ \\
\hline
\end{tabular}

Fonte: Autores (2020).

\section{Discussão}

No ano de 2014 foi identificado um número menor de notificações do que o esperado, provavelmente por ainda não haver uma cultura de segurança implantada no ambiente de trabalho. Foi observado um aumento da adesão ao processo, totalizado 5.488 notificações quando analisamos por metas e 5.344 quando observados os tipos de ocorrência, entre 2014 e 2019.

Foi observado que entre as metas, a mais notificada foi a Meta 1, relacionada a erros na identificação do paciente, totalizando 3.636 notificações $(66,20 \%)$. Pode-se perceber que os anos 2015, 2016 e 2017 os números relacionados a essa notificação eram bem altos, 979 (15,13\%), 1.186 (17,33\%) e 878 (12,63\%) respectivamente e nos anos de 2018 e 2019 esses números reduziram consideravelmente para 266 (2.64\%) e $323(2,96 \%)$. Isso ocorreu, provavelmente, por maior conscientização dos profissionais de saúde sobre a relevância em identificar os pacientes. Os métodos tradicionais de identificação do paciente incluem a verificação do nome, data de nascimento ou número do paciente fornecido pelo hospital. Os incidentes que prejudicam o paciente por erros de identificação são considerados evitáveis. Um hospital universitário em Porto Alegre no RS com 841 leitos avaliou uma amostra de 385 internações e observou que 46 (11,9\%) pacientes estavam identificados em sem conformidade com o Procedimento Operacional Padrão de Identificação, enquanto 16 (4,2\%) pacientes não estavam identificados. (Hoffmeister, 2015). Esse percentual de notificação foi semelhante ao observado no período de 2015 a 2017 do nosso estudo.

Em um grande hospital terciário canadense, mais de $6 \%$ dos pacientes cirúrgicos tiveram suas pulseiras de identificação removidas, geralmente para o acesso vascular quando já estão na sala de cirurgia, correndo o risco de haver esquecimento em substituí-las, o que acontece em 60\% dos casos (Christopher, 2017). Uma contribuição importante nesse estudo é a reflexão sobre os incidentes de identificação que ocorrem em vários âmbitos e devem ser contemplados para reduzilos ao máximo. Uma alternativa testada foi a implantação de reconhecimento facial demonstra ser um método de verificação adequado, com precisão de 99\% (Jeon, 2019).

Os incidentes relacionados à comunicação efetiva, Meta 2, resultaram em 192 (3,50\%) no período de 2014 a 2019. É 
possível que exista subnotificação desses dados já que a comunicação verbal e escrita perpassam processos assistenciais e administrativos. Nos Estados Unidos, erros de comunicação foram identificados como a causa raiz de 1.796 eventos sentinela nos anos 2013 a 2015 como um fator causador de atrasos no tratamento, medicamentos e procedimentos incorretos (The Joint Commission, 2016). No Japão, falha de comunicação foi identificada em 524 eventos adversos entre 2010 e 2017 (Conselho do Japão para Cuidados em Saúde, 2017). Na região Sul do Brasil foi observado que o uso do Modified Early Warning Score (MEWS), assegurou comunicação e seguimento do cuidado do paciente após transferência, com mais de $80 \%$ de adesão à ferramenta (Olino, 2019).

As notificações relacionadas à Meta 3, no processo de prescrição, distribuição, dispensação, preparação e administração de medicamentos, foram de 204 (3,71\%) no período entre 2014 e 2019. O custo mundial estimado dos erros de medicação é de US\$ 42 bilhões anualmente. A WHO lançou em 29 de março de 2017 uma iniciativa global para reduzir em $50 \%$ os danos graves e evitáveis associados a medicamentos em todos os países nos cinco anos subsequentes (WHO, 2017).

A Segurança nas Cirurgias, Meta 4, neste estudo, foram registradas 31 (0,56\%) notificações com entre 2014 a 2019. É possível que os eventos tenham sido subnotificados devido à resistência de alguns colaboradores, essa tendência foi observada em dados referentes ao Brasil e ao Rio de Janeiro, segundo o relatório de incidentes notificados pelos NSP e por meio do Notivisa. Entre os anos 2019 e 2020, no Brasil, observou-se aproximadamente 1000 falhas em procedimentos cirúrgicos e no Estado do Rio de Janeiro, foram 25 registros, conforme os dados do sistema Notivisa. As complicações cirúrgicas representam importante causa de invalidez e morte, com consequências significativas na Saúde Pública (WHO, 2009). Cerca de 15\% dos pacientes que se submetem à cirurgia apresentam eventos adversos (Anderson, 2013). A lista de verificação de segurança nas cirurgias (checklist), relacionadas ao paciente e ao procedimento, revisa pontos críticos e itens a serem cuidadosamente analisados, com intuito de se praticar intervenção cirúrgica segura (WHO, 2009).

Em relação à Meta 5, foram registrados 740 (13,47\%) infecções associadas ao cuidado em saúde, de 2014 a 2019. Um estudo brasileiro realizado em 2018 caracterizou os pacientes quanto aos eventos adversos infecciosos mais frequentes ocorridos em 182 hospitais situados em 13 estados distintos; o número de notificações por ano e por hospital foi em média de 19,5\%. Foram identificados 1.210 (15\%) pacientes com infecção do trato urinário; 952 (11.8\%) pacientes com septicemia; 831 (10.3\%) pacientes com infecção subsequente a algum procedimento e 561(7.0\%) com pneumonia bacteriana. Os dados utilizados foram coletados por meio de altas hospitalares codificadas entre 01/04/2017 a 31/03/2018 pelas operadoras de saúde suplementar, gestores do SUS na rede hospitalar credenciada e por hospitais que utilizam a metodologia DRG Brasil (Diagnosis Related Groups-Universidade do Yale), (Couto, 2018).

Observamos no estudo da Meta 6, um total de 220 (4,00\%) notificações referentes à queda de paciente, entre $2014 \mathrm{e}$ 2019, esses dados foram inferiores do que o esperado. No Brasil, entre 2019 e 2020, os incidentes relacionados à quedas foram próximos a 12.000 e no Estado do Rio de Janeiro, em torno de 875 incidentes (Notivisa, 2019).

O estudo da Meta 7 registrou $77(1,40 \%)$ notificações de úlceras de pressão. Esses números observados não corresponderam ao resultado esperado, estando provavelmente subnotificados. No Brasil, de abril de 2019 a março de 2020, foram observados 30.000 incidentes referentes a úlceras por pressão, representando o segundo lugar de todos os incidentes. No Estado do Rio de Janeiro, as úlceras por pressão chegaram a 600 notificações aproximadamente (Notivisa, 2019).

Neste estudo, o total de eventos adversos notificados em seis anos foi de 1.140 (21,33\%), porém se observarmos os dados dos anos de 2017, 2018 e 2019 em que o conhecimento sobre a segurança estava mais solidificado, os eventos adversos foram de 260 notificações (3,74\%), 315 notificações (3,13\%), 456 notificações $(4,17 \%)$, respectivamente, se aproximando do intervalo observado nos países desenvolvidos que varia de $4 \%$ a $16 \%$ dos pacientes hospitalizados (ANVISA, 2019). Países membros da União Europeia demonstram ocorrência de 8 a 12\% de eventos adversos nas internações (WHO, 2018), já no Brasil, a prevalência de eventos adversos em pacientes internados no SUS é de 6.4\%, sendo 1,9\% de eventos adversos graves e 
$22,8 \%$ de mortalidade. No SSUP a prevalência de eventos adversos é 7,1\%, sendo 1,4\% de eventos adversos graves e com mortalidade de $12 \%$ (Couto, 2018).

A ocorrência de notificações para incidentes sem dano foi de 3.029 (56,68\%), com números mais elevados de 2015 a 2017, reduzindo consideravelmente nos anos 2018 e 2019, respectivamente: 1,44\% e 1,02\%. Conforme base de dados da Inglaterra, o "National Reporting and Learning System (NRLS)", os incidentes sem dano entre outubro de 2017 e setembro de 2019 atingiram 73,65\%, ou seja, quase 3 quartos dos incidentes, notificadosnum total de 5.879 .954 relatórios de incidentes coletados de hospitais ao longo de décadas, sendo que cerca de 70,3\% dos incidentes não produziram danos ao paciente (NRLS, 2015).

Em relação às circunstâncias notificáveis, foram registradas 883 (16,52\%) notificações, são situações potencialmente perigosas quépodem levar a sérias consequências. Em estudo descritivo transversal, retrospectivo em um hospital de ensino em Goiás foram identificados 2.718 incidentes do tipo circunstância notificável, média de 453 por ano ou 37,7 por mês, ocorridos na unidade de clínica médica (Sagawa, 2019).

Em relação à quase falhas, significa um incidente que foi detectado antes de ocorrer. Neste estudo foram notificadas quase falhas em 292 (5,46\%). Estudo retrospectivo descritivo de incidentes de quase falha registrados no sistema de relatório, em hospital de grande porte no sul do país com 497 leitos, identificou um total de 12.939 quase falhas registradas entre 2013 e 2017, com crescimento linear. A near miss foi mais frequentes para medicamentos, processos não especificados e por controle de prontuários, balanço hídrico, punção venosa (Lorenzini, 2020).

\section{Conclusão}

Caracterizar e analisar os dados relacionados aos incidentes ocorridos no hospital é um exercício fundamental pois auxilia o processo de educação permanente sobre segurança do paciente, determinação de protocolos, treinamentos, formação de equipes especializadas, facilidade no acesso às notificações e consequentemente maior aprimoramento no cuidado seguro aos pacientes. Nesse sentido, o presente estudo contribui ao evidenciar a importância da implementação de esforços em prol da melhoria da qualidade, como por exemplo com a constituição do Núcleo de Segurança do Paciente (NSP), que impactou no aumento das notificações ao organizar os processos de registro. Além disso, podemos observar que permanecem como desafios a alta incidência de falha na identificação correta do paciente (Meta 1) e a prevenção de infecções (Meta 5), remetendo a necessidade de melhorias no processo de educação permanente e adesão dos profissionais envolvidos, bem como na manutenção do investimento no monitoramento dos eventos no âmbito hospitalar. Futuros estudos devem avaliar o impacto das ações desenvolvidas pelo Núcleo, bem como a evolução da adesão dos profissionais e os dados de monitoramento de eventos com vistas a compreender o status da questão no Hospital.

\section{Referências}

Anderson, O. S. et al. (2013). Surgical adverse events: a systematic review. Am. J Surg. 206(2), 253-62.

ANVISA. (2019). Relatório de Autoavaliação Nacional das Práticas de Segurança do Paciente em Serviços de Saúde. Agência Nacional de Vigilância Sanitária (ANVISA). Ministério da Saúde.

ANVISA. (2020). Sistema Nacional de Notificações em Vigilância Sanitária: Módulo Saúde 2020. Agência Nacional de Vigilância Sanitária (ANVISA). Ministério da Saúde.

Brasil. (2009). Portaria n 1660 do Ministério da Saúde, de 22 de julho de 2009, instituiu o Sistema de Notificação e Investigação em Vigilância Sanitária, no âmbito do Sistema Nacional de Vigilância Sanitária. Ministério da Saúde.

Brasil. (2009). Portaria n 529 do Ministério da Saúde de 01 de abril de 2013, instituiu o Programa Nacional de Segurança do Paciente. Ministério da Saúde.

Couto, R. C. et al.(2018). II Anuário da Segurança Assistencial Hospitalar No Brasil. Propondo as prioridades nacionais. Minas Gerais, Brasil: Instituto de Estudos de Saúde Suplementar, 2018. 
Research, Society and Development, v. 10, n. 4, e30510414080, 2021

(CC BY 4.0) | ISSN 2525-3409 | DOI: http://dx.doi.org/10.33448/rsd-v10i4.14080

Comission, J. Sentinel event statistics data - Event type by year (1995-Q2-2016).

Christopher L. Pysky et al. (2017). A change to the surgical safety checklist to reduce patient identification erros. Ottawa, Canadá: Department of Anesthesiology and Pain Medicine, The Ottawa,Hospital, Faculty of Medicine, University of Ottawa, Ottawa

Institute of Medicine. (2001). Crossing the quality chasm: A new health care system for the 21st century. Japan Council for Quality Health Care, 2017. Washington, DC: National Academy.

Joint Commission for Patient Safety (2008). World Alliance for Patient Safety.

Jeon. B. S. et al (2019). A facial recognition mobile app for patient safety and biometric identification: design, development, and validation. byoungiun. JMIR Mhealth and Uhealth. 7(4). 8. 2019).

Hoffmeister, V. et al. (2015). Uso de pulseiras de identificação em pacientes internados em um hospital universitário. Revista Latino-Americana de Enfermagem. Universidade de São Paulo.

Lorenzini, E. et al. (2020). Near-miss analysis in a large hospital in southern Brazil: A 5-year retrospective study. The International Journal of Risk \& Safety in Medicine.

NHS. (2018). NRLS. National patient safety incident reports: commentary.

NIHR, (2016). Patient safety translational research centre, Imperial College London. National reporting and learning system research and development. London: Imperial College Healthcare; 2016

Olino. L. et al. (2019). Comunicação efetiva para a segurança do paciente: nota de transferência e aviso prévio modificado. Revista Gaúcha de Enfermagem Porto Alegre, Brasil.

Sagawa, M. R., et al. (2019). Análise de circunstâncias notificáveis: incidentes que podem comprometer a segurança dos pacientes. Revista Cogitare Enfermagem. Brasil, Paraná: Universidade Federal do Paraná.

Slawomirski, L, Auraaen, A. \& Klazinga, N. (2017). The economics of patient safety: strengthening a value-based approach to reducing patient harm at national level. (pp. 23). Paris, França: Organisation for Economic Co-operation and Development.

Walter, M. (2019). Taxonomia em Segurança do paciente: Sousa, P.; Mendes, W. (org.). Segurança do paciente. Conhecendo os riscos nas organizações de saúde. Fundação Oswaldo Cruz - Escola Nacional de Saúde Pública Sergio Arouca

World Health Organization. (2008). Forward programme 2008-2009. World Alliance for Patient Safety.

World Health Organization. (2009). Global Priorities for Patient Safety Research.

World Health Organization. (2009).Safety checklist, patient safety. A World Alliance for Safer Health Care.

World Health Organization. (2009). The conceptual framework for the International classification for patient safety: final technical report. World Alliance for Patient Safety.

World Health Organization. (2012). Research introductory course: What is patient safety. pp. 02. World Alliance for Patient Safety.

World Health Organization. (2017). Challenge on medication safety. Global Patient Safety. 